Mathematical Research Letters 8, 509-519 (2001)

\title{
NON-PAC FIELDS WHOSE HENSELIAN CLOSURES ARE SEPARABLY CLOSED
}

\author{
Wulf-Dieter Geyer and Moshe Jarden
}

\section{Introduction}

A field $K$ is $P A C$ if every nonvoid absolutely irreducible variety $V$ over $K$ has a $K$-rational point. The concept of a PAC field originated in Ax' work [Ax] on the elementary theory of finite fields. Although finite fields are not PAC, nonprincipal ultra products of finite fields (Ax, [FrJ, Cor. 10.6]) and infinite algebraic extensions of finite fields (Ershov, [FrJ, Cor. 10.7]) are PAC. Each separably closed field is PAC. If $K$ is a countable Hilbertian field and $e$ is a positive integer, then for almost all $\boldsymbol{\sigma} \in G(K)^{e}$ the field $K_{s}(\boldsymbol{\sigma})$ is PAC [FrJ, Thm. 16.18]. Here $G(K)$ is the absolute Galois group of $K$ equipped with the Haar measure, $K_{s}$ is the separable closure of $K$, and $K_{s}(\boldsymbol{\sigma})$ is the fixed field of $\sigma_{1}, \ldots, \sigma_{e}$ in $K_{s}$. An explicit example of an algebraic extension of $\mathbb{Q}$ which is PAC is $\mathbb{Q}_{\operatorname{tr}}(\sqrt{-1})$, where $\mathbb{Q}_{\operatorname{tr}}$ is the field of all totally real algebraic numbers (Pop). Finally, Fried starts from an arbitrary field $K_{0}$ and adjoins algebraically independent generic points of all absolutely irreducible varieties over $K_{0}$. Then he iterates this construction inductively. Finally he takes the union of the sequence of fields obtained in this way to obtain a regular extension $K$ of $K_{0}$ which is PAC [FrJ, Prop. 12.11].

Although one expects most fields to be non-PAC, it is not easy to construct one. Clearly, if $K$ is formally real, then it is non-PAC [FrJ, Thm. 10.2]. If $K$ has a valuation $v$ whose residue field is finite, then $K$ is non-PAC (Ax). More generally, if the Henselian closure $K_{v}$ of $K$ with respect to $v$ is not separably closed, then $K$ is non-PAC (Frey-Prestel [FrJ, Thm. 10.14]). Consequently, [FrJ, Prob. 10.16(b)] raises the following problem:

Problem A. Is there an infinite field $K$ which is neither formally real nor PAC all of whose Henselian closures are separably closed?

A recent work of Efrat gives a clue to the solution of Problem A. To this end consider a field $K$ and let $F$ be an extension of $K$ of transcendence degree 1 . Denote the set of all equivalence classes of valuations of $F$ which are trivial on $K$ by $\mathbb{P}(F / K)$. For each $\mathfrak{p} \in \mathbb{P}(F / K)$ let $F_{\mathfrak{p}}$ be the Henselian closure of $F$ at $v$.

Received January 9, 2001.

Research supported by the Minkowski Center for Geometry at Tel Aviv University 
We say that $F$ satisfies the Hasse principle for Brauer groups if the restriction maps of Brauer groups

$$
\text { res: } \operatorname{Br}(F) \rightarrow \prod_{v \in V(F / K)} \operatorname{Br}\left(F_{v}\right)
$$

is injective.

Proposition B. Let $K$ be a perfect field.

(a) If $K$ is PAC, then each extension $F$ of $K$ of transcendence degree 1 satisfies the Hasse principle for Brauer groups [Efr, Thm. 3.4].

(b) Suppose that $K$ is not necessarily PAC but each extension $F$ of $K$ of transcendence degree 1 satisfies the Hasse principle for Brauer groups. Then every nontrivial valuation of $K$ has an algebraically closed residue field and a divisible value group [Efr, Thm. 4.1].

Following these results, Efrat [Efr, Question 4.2] asks:

Problem C. Let $K$ be a non-real infinite perfect field such that the Hasse principle holds for all extensions $F$ of $K$ of relative transcendence degree 1 . Is $K$ necessarily PAC?

The goal of this work is to construct a field which simultaneously solves both Problems.

Theorem D. Let $K_{0}$ be either a finite field or a global field. Then $K_{0}$ has an infinite regular extension $K$ with the following properties:

(a) Every extension $F$ of $K$ of transcendence degree 1 satisfies the Hasse principle for Brauer groups.

(b) $K$ is not formally real.

(c) Each Henselization of $K$ is separably closed.

(d) $K$ is not PAC.

Our construction follows that of Fried which we mentioned above. However instead of adjoining generic points of all varieties we adjoin only generic points of varieties which are birationally equivalent over the algebraic closure to either a rational variety or to an abelian variety. If the basic field $K_{0}$ is either a finite field or a number field, then the constructed field $K$ is not PAC but each variety of the above types has a $K$-rational point. So, $K$ is weakly PAC. Using the results of [Efr], we prove that $K$ satisfies conditions (a)-(d) of Theorem D.

\section{Weakly PAC fields}

Let $K$ be a field. Denote the algebraic (resp., separable, purely inseparable) closure of $K$ by $\tilde{K}$ (resp., $K_{s}, K_{\text {ins }}$ ) and let $G(K)=\mathcal{G}\left(K_{s} / K\right)$ be the absolute Galois group of $K$. When we say that $V$ is a variety (or a curve) over $K$ we mean that $V$ is absolutely irreducible and nonempty. This is the case if $V$ is 
irreducible and if the function field of $V$ is a regular extension of $K$ [FrJ, Lemma 9.5]. If $L$ is an extension of $K$, we abbreviate $V \times_{K} L$ by $V_{L}$ and put $\tilde{V}=V_{\tilde{K}}$. Similarly, if $\alpha: V \rightarrow W$ is a rational map, then $\tilde{\alpha}: \tilde{V} \rightarrow \tilde{W}$ is the rational map which is obtained from $\alpha$ by extension of scalars from $K$ to $\tilde{K}$.

Recall that $K$ is $P A C$ if each variety $V$ over $K$ has a $K$-rational point. In this work we impose the latter condition only on varieties of restrictive type. We obtain "weakly PAC fields" which are not always PAC fields.

Let $V$ be a variety over $K$. We say that $V$ is a variety of type $i, i=0,1$, if the following condition holds:

(Type 0) $\tilde{V}$ is birationally equivalent to $\mathbb{A}^{n}$ for some positive integer $n$.

(Type 1) $\tilde{V}$ is birationally equivalent to an abelian variety of positive dimension.

Here are some simple conservation rules for the types that follow immediately from the definition:

(1a) Suppose that $V$ and $V^{\prime}$ are birationally equivalent varieties over $K$. If $V$ is of type $i$, then so is $V^{\prime}$.

(1b) If $V_{1}, \ldots, V_{m}$ are varieties over $K$ of type $i$, then so is $V_{1} \times \cdots \times V_{m}$.

(1c) If $V$ is a variety over $K$ of type $i$, then so is $V_{L}$ for every field extension $L$ of $K$.

(1d) Suppose that $V$ is a variety over $K, L$ is an algebraic extension of $K$, and $W$ is a variety of type $i$ over $L$ which is birationally equivalent to $V_{L}$. Then $V$ is of type $i$.

We say that $K$ is weakly $P A C$ if each affine variety $V$ over $K$ of type 0 or 1 has a $K$-rational point. It follows from (1a) that $V(K)$ is Zariski-dense in $V$ for each projective variety of type 0 or 1 over $K$. In particular, $K$ is an infinite field. By definition, each PAC field is weakly PAC.

Lemma 1.1. Let $K$ be a field and let $L$ be an algebraic extension of $K$.

(a) If each variety over $K$ of type $i$ has a $K$-rational point, then each variety over $L$ of type $i$ has a $K$-rational point, $i=0,1$.

(b) If $K$ is weakly PAC, then so is $L$.

Proof. Condition (b) follows from condition (a). In order to prove (a), we may assume that $L$ is a finite extension of $K$. Moreover, we may assume that either $L$ is a separable extension of $K$ or $L$ is a purely inseparable extension of $K$. Consider therefore a variety $V$ over $L$ of type $i$.

If $L / K$ is separable, then Weil's descent gives an affine variety $W$ over $K$ such that $\tilde{W} \cong \tilde{V}^{d}$ and a morphism $\alpha: W_{L} \rightarrow V$ [FrJ, Prop. 9.34].

If $L / K$ is purely inseparable, then a theorem of Roquette gives a variety $V$ over $K$ and a birational morphism $\alpha: W_{L} \rightarrow V$ [FrJ, Prop. 9.35].

Rules (1a)-(1d) imply that $W$ is of type $i$. By assumption, $W$ has a $K$-rational point. Hence, $V$ has an $L$-rational point.

J. Ax observes in [Ax, p. 269, Lemma 2] that if $K$ is a PAC field, then its Brauer group $\operatorname{Br}(K)$ is trivial. The proof uses the fact that the reduced norm of 
a simple central $K$-algebra is an absolutely irreducible homogeneous polynomial. As $K$ is PAC, this polynomial has a nontrivial $K$-rational zero. This implies that $A$ splits over $K$.

For weakly PAC field we have to use an alternative proof. It reproves $\mathrm{Ax}$ ' result.

Proposition 1.2. Let $K$ be a field. Suppose that each variety over $K$ of type 0 has a $K$-rational point. Then $\operatorname{Br}(K)=0$ and $G(K)$ is projective. In particular, this holds if $K$ is weakly PAC.

Proof. Each simple central $K$-algebra $A$ of dimension $n^{2}$ corresponds to a SeveriBrauer variety $V$ over $K$ of dimension $n-1$ [Ser, P. 168]. By definition, $\tilde{V} \cong$ $\mathbb{P}^{n-1}$. In particular, $V$ is a variety of type 0 . By assumption, $V$ has a $K$ rational point. Hence, $A$ splits over $K$ [Jac, p. 113, Thm. 3.5.6]. Conclude that $\operatorname{Br}(K)=0$.

By Lemma 1.1, each algebraic extension $L$ of $K$ is weakly PAC. By the preceding paragraph, $\operatorname{Br}(L)=0$. It follows that $\operatorname{cd}(G(K)) \leq 1$ [Rib, p. 262, Cor. 3.8]. In other words, $G(K)$ is projective.

Let $F$ be an extension of a field $K$. A prime divisor of $F / K$ is an equivalence class of valuations of $F$ which are trivial over $K$. We denote the set of all prime divisors of $F / K$ by $\mathbb{P}(F / K)$. For each $\mathfrak{p} \in \mathbb{P}(F / K)$ we denote the Henselian closure of $F$ with respect to $\mathfrak{p}$ by $F_{\mathfrak{p}}$. It is unique up to a $K$-isomorphism. Tensoring central simple finite dimensional $F$-algebras with $F_{\mathfrak{p}}$ defines a homomorphism res $\mathfrak{p}_{\mathfrak{p}} \mathrm{Br}(F) \rightarrow \operatorname{Br}\left(F_{\mathfrak{p}}\right)$. We consider the direct product of all these homomorphisms:

$$
\text { res: } \operatorname{Br}(F) \rightarrow \prod_{\mathfrak{p} \in \mathbb{P}(F / K)} \operatorname{Br}\left(F_{\mathfrak{p}}\right)
$$

and say that $F$ satisfies the Hasse principle for Brauer groups if res is injective.

Ido Efrat [Efr, Thm. 3.4] proves that if $K$ is a perfect PAC field, then each extension $F$ of $K$ of transcendence degree 1 satisfies the Hasse principle for Brauer groups. A careful analysis of Efrat's proof shows that it works even if $K$ is perfect and weakly PAC [Efr, Remark 3.5(c)].

Proposition 1.3. Let $K$ be a weakly PAC field. Then

(a) each extension $F$ of $K_{\text {ins }}$ of transcendence degree 1 satisfies the Hasse principle for Brauer groups,

(b) all Henselian closures of $K$ are separably closed, and

(c) $K$ is not formally real.

Proof of $(a)$. By Lemma 1.1, $F \cap \tilde{K}$ is a perfect weakly PAC field. Replace $K$ by $F \cap \tilde{K}$, if necessary, to assume that $K$ is perfect and $F / K$ is a regular extension. By [Efr, Lemma 3.3], we may assume that $F / K$ is finitely generated. 
In other words, $F$ is a function field of one variable over $K$. As $\operatorname{cd}(G(K)) \leq 1$ (Proposition 1.2) there is an exact sequence

$$
0 \rightarrow H^{1}(G(K), \operatorname{Pic}(F \tilde{K} / \tilde{K})) \rightarrow \operatorname{Br}(F) \stackrel{\text { res }}{\longrightarrow} \bigoplus_{\mathfrak{p} \in \mathbb{P}(F / K)} \operatorname{Br}\left(F_{\mathfrak{p}}\right)
$$

where $\operatorname{Pic}(F \tilde{K} / \tilde{K})$ is, as usual, the quotient group of all divisors of $F \tilde{K} / \tilde{K}$ modulo principal divisors [Efr, Prop. 2.3]. Denote the Jacobian of $F / K$ by $J$. By [Efr, Lemma 1.4] there is a natural epimorphism

$$
H^{1}\left(G(K), J\left(K_{s}\right)\right) \mapsto H^{1}(G(K), \operatorname{Pic}(F \tilde{K} / \tilde{K}))
$$

Each element of $H^{1}\left(G(K), J\left(K_{s}\right)\right)$ may be represented by a variety $V$ over $K$ such that $\tilde{V} \cong \tilde{J}$. Since $K$ is weakly-PAC, $V$ has a $K$-rational point. Hence, $V$ represents the trivial element of $H^{1}\left(G(K), J\left(K_{s}\right)\right)$ [LaT, Prop. 4]. It follows that $H^{1}\left(G(K), J\left(K_{s}\right)\right)=0$. Hence, $H^{1}(G(K)$, Pic $(F \tilde{K} / \tilde{K})=0$. Conclude from (3) that res: $\left.\operatorname{Br}(F) \rightarrow \prod_{\mathfrak{p} \in \mathbb{P}(F / K)} \operatorname{Br}\left(F_{\mathfrak{p}}\right)\right)$ is injective.

Proof of $(b)$. Let $v$ be a valuation of $K$. Denote the unique extension of $v$ to $K_{\text {ins }}$ by $v_{\text {ins. }}$. Let $v_{s}$ be an extension of $v$ to $K_{s}$ and let $\tilde{v}$ be the unique extension of $v_{s}$ to $\tilde{K}$. Then the isomorphism res: $G\left(K_{\text {ins }}\right) \rightarrow G(K)$ maps the decomposition group $D(\tilde{v})=\left\{\sigma \in G\left(K_{\text {ins }}\right) \mid \tilde{v} \circ \sigma=\tilde{v}\right\}$ of $\tilde{v}$ onto the decomposition group $D\left(v_{s}\right)=\left\{\sigma \in G(K) \mid v_{s} \circ \sigma=v_{s}\right\}$ of $v$. So, if we denote the fixed field of $D\left(v_{s}\right)$ by $K_{v}$, we find that the fixed field of $D(\tilde{v})$ is $K_{v} K_{\text {ins }}$, which is $\left(K_{v}\right)_{\text {ins. }}$. So, $K_{v}$ is a Henselian closure of $(K, v)$ and $K_{v} K_{\text {ins }}$ is a Henselian closure of $\left(K_{\text {ins }}, v_{\text {ins }}\right)$. By Lemma $1.1, K_{\text {ins }}$ is weakly PAC. Hence, replacing $K$ by $K_{\text {ins }}$, if necessary, we may assume that $K$ is perfect.

Since the Henselian closure of $K$ with respect to $v$ is also weakly PAC (Lemma $1.1)$, we may as well assume that $(K, v)$ is Henselian. By (a) and by [Efr, Thm. 4.1] the residue field $\bar{K}_{v}$ is separably closed and the value group $\Gamma_{v}$ is divisible. As [Efr, Thm. 4.1] points out, if $\operatorname{char}\left(\bar{K}_{v}\right)=0$, this implies that $\operatorname{char}(K)=0$ and that $K$ is algebraically closed. We prove that for an arbitrary $v, K$ is separably closed.

Suppose first that $\operatorname{rank}(v)=1$. Then $v$ is in the terminology of [Fre], a real non-Archimedean valuation. If $K$ were not separably closed we could choose a nontrivial element $\sigma$ of $G(K)$ and use Lemma 1.1 again to replace $K$ by the fixed field of $\sigma$ in $K_{s}$. Thus, we could assume without loss that $G(K)$ is procyclic. But then, by [Fre, p. 205, Lemma], there would exist a curve $E$ over $K$ of genus 1 without $K$-rational points. As $\tilde{E}$ is an elliptic curve, that is, an abelian variety of dimension 1, this would contradict the assumption that $K$ is a weakly PAC field.

In the general case we consider the set of all nontrivial valuation rings that contain $O_{v}$. It is indexed by a totally ordered set $I$ such that if $i \leq j$, then $O_{j} \subseteq O_{i}$ and $\mathfrak{m}_{i} \subseteq \mathfrak{m}_{j}$, where $\mathfrak{m}_{i}$ is the maximal ideal of $O_{i}$ [Rib, Chap. C]. By 
[Rib, p. 210, Prop. 9], each $O_{i}$ is a Henselian valuation ring. Hence, by Efrat's result mentioned in the second paragraph, its residue field $\bar{K}_{i}$ is separably closed.

The ring $O=\bigcup_{i \in I} O_{i}$ is either a valuation ring or $O=K$. If $O$ is a valuation ring, then it is a maximal one. In other words, $O$ has rank 1. By Frey's lemma, $K$ is separably closed.

Assume therefore that $O=K$. Let $f \in K[X]$ be a monic irreducible separable polynomial. Then its discriminant $d$ is nonzero. Also, there exists $j$ such that $f \in O_{j}[X]$. Hence, $f \in O_{i}[X]$ and $d \in O_{i}$ for all $i \leq j$. Since in our case, $\bigcap_{i \leq j} \mathfrak{m}_{i}=0$, there exists $k \leq i$ such that $d \notin \mathfrak{m}_{k}$. Then the residue of $d$ modulo $\mathfrak{m}_{k}$ is not zero. This means that $f$ has no multiple roots modulo $\mathfrak{m}_{k}$. As $O_{k} / \mathfrak{m}_{k}$ is separably closed (by the first paragraph), $f$ has a simple zero modulo $\mathfrak{m}_{k}$. Since $O_{k}$ is Henselian, this zero can be lifted to a zero of $f$ in $O_{k}$. Conclude that $K$ is separably closed.

Proof of $(c)$. Assume without loss that $\operatorname{char}(K)=0$. Consider the conic $C$ defined over $K$ by the equation $X^{2}+Y^{2}+1=0$. Its extension $\tilde{C}$ to $\tilde{K}$ is a rational curve [Art, p. 304]. Hence $C$ is of type 0 and therefore has a $K$-rational point. It follows that $K$ has no ordering. In other words, $K$ is not formally real (Indeed, its level is at most 2.)

\section{Examples}

We construct weakly PAC fields which are not PAC. By Proposition 1.3(b,c), this gives a negative answer to Problem C. By Proposition 1.3(d), this also solves Problem A. The construction depends on a lemma which handles varieties of somewhat more general types than those of Section 1. We say that a variety $V$ over a field $K$ is of type $i^{\prime}, i=1,2$, if the following conditions hold.

(Type $0^{\prime}$ ) There exists a dominating rational map $\alpha: \mathbb{A}^{n} \rightarrow \tilde{V}$, for some positive integer $n$. That is, $\tilde{V}$ is unirational.

(Type $1^{\prime}$ ) There exists an algebraic group $H$ over $\tilde{K}$ and a dominating rational $\operatorname{map} \alpha: H \rightarrow \tilde{V}$.

In particular, if $V$ is of type $i$, then it is of type $i^{\prime}$. The following lemma justifies the terminology.

Lemma 2.1. Let $C$ be a curve over a field $K$ and let $V$ be a variety over $K$. Denote the function field of $V$ over $K$ by $F$. Suppose that $C(F) \neq C(K)$.

(a) If $V$ is of type $0^{\prime}$, then genus $(\tilde{C})=0$.

(b) If $\tilde{V}$ of type $1^{\prime}$, then genus $(\tilde{C}) \leq 1$.

Proof. We choose a point $\mathbf{p} \in C(F) \backslash C(K)$. It is a generic point of $C$ over $K$. The rest of the proof breaks up into two parts.

Proof of $(a)$. Suppose that $\tilde{V}$ is unirational. Then $F \tilde{K}$ is contained in a purely transcendental extension $\tilde{K}\left(x_{1}, \ldots, x_{n}\right)$ of $\tilde{K}$. Hence $\tilde{K}(\mathbf{p}) \subseteq \tilde{K}\left(x_{1}, \ldots, x_{n}\right)$. Since trans. $\operatorname{deg}(\tilde{K}(\mathbf{p}) / \tilde{K})=1$, a theorem of Lüroth-Gordan-Igusa implies that 
$\tilde{K}(\mathbf{p})=\tilde{K}(t)$, with $t$ transcendental over $K$ [Sch, p. 9]. Hence it follows that $\operatorname{genus}(\tilde{C})=0$.

Proof of $(b)$. Suppose there exists an algebraic group $H$ and a dominating rational map $\alpha: H \rightarrow \tilde{V}$. The inclusion $K(\mathbf{p}) \subseteq F$ defines a dominating rational map $\beta: V \rightarrow C$ over $K$. Assume that genus $(\tilde{\tilde{C}})>0$ and let $\gamma: \tilde{C} \rightarrow J$ be an embedding of $\tilde{C}$ into its Jacobian $J$. Then $\theta=\gamma \circ \tilde{\beta} \circ \alpha$ is a rational map from $H$ into $J$ which is defined over $\tilde{K}$. By [Lan1, p. 24, Thm. 4], $J$ has an abelian subvariety $\Gamma$ and there is $\mathbf{a} \in A(\tilde{K})$ such that $\theta(H(\tilde{K}))=\mathbf{a}+\Gamma(\tilde{K})$. On the other hand, $\gamma$ is a birational map between $\tilde{C}$ and $\theta(H)$. It follows that $\operatorname{dim}(\Gamma)=1$. Conclude that $\operatorname{genus}(\tilde{C})=\operatorname{genus}(\Gamma)=1$.

Corollary 2.2. Let $V=V_{1} \times V_{2} \times \cdots \times V_{n}$ be a direct product of varieties over a field $K$. Suppose that each $V_{i}$ is either of type $0^{\prime}$ or of type $1^{\prime}$. Denote the function field of $V$ over $K$ by $F$. Then $C(F L)=C(L)$ for each algebraic extension $L$ of $K$ and for each curve $C$ over $L$ with genus $(\tilde{C}) \geq 2$.

Proof. If $L$ is an algebraic extension of $K$, then $F L$ is the function field of $C \times{ }_{K} L$. So, without loss, we may consider a curve $C$ over $K$ such that genus $(\tilde{C}) \geq 2$ and prove that $C(F)=C(K)$.

Indeed, $F=K\left(\mathbf{x}_{1}, \mathbf{x}_{2}, \ldots, \mathbf{x}_{n}\right)$, where $\mathbf{x}_{i}$ is a generic point of $V_{i}$ over $K$, $i=1, \ldots, n$ and $K\left(\mathbf{x}_{1}\right), K\left(\mathbf{x}_{2}\right), \ldots, K\left(\mathbf{x}_{n}\right)$ are algebraically independent (i.e, free) over $K$. For each $m$ between 0 and $n$ let $F_{m}=K\left(\mathbf{x}_{1}, \ldots, \mathbf{x}_{m}\right)$. Let $m<n$ and inductively assume that $C\left(F_{m}\right)=C(K)$. Then $F_{m+1}=F_{m}\left(\mathbf{x}_{m+1}\right)$ is the function field of the variety $V_{m+1} \times_{K} F_{m}$ which is either of type $0^{\prime}$ of type $1^{\prime}$. Hence, by Lemma 2.1, $C\left(F_{m+1}\right)=C\left(F_{m}\right)$. So, $C\left(F_{m+1}\right)=C(K)$. Conclude by induction that $C(F)=C(K)$.

Lemma 2.3. Let $K_{0} \subseteq K_{1} \subseteq K_{2} \subseteq \cdots$ be an ascending sequence of fields such that $K_{j}$ is (separably) Hilbertian and $K_{j+1}$ is a regular extension of $K_{j}$, $j=1,2,3, \ldots$. Then $K=\bigcup_{j=0}^{\infty} K_{j}$ is a (separably) Hilbertian field.

Proof. Consider irreducible (separable) polynomials $f_{1}, \ldots, f_{m} \in K[T, X]$. Then there exists $j \geq 1$ such that $f_{1}, \ldots, f_{m} \in K_{j}[T, X]$. Hence, there exists $a \in K_{j}$ such that for each $i$ the polynomial $f_{i}(a, X)$ is irreducible over $K_{j}$. Let $x_{i}$ be a root of $f_{i}(a, X)$. By assumption $K$ is a regular extension of $K_{j}$. In particular, $K$ is linearly disjoint from $K_{j}\left(x_{1}, \ldots, x_{m}\right)$ over $K_{j}$. Hence, $f_{i}(a, X)$ is irreducible over $K, i=1, \ldots, m$. Conclude that $K$ is (separably) Hilbertian.

A finite embedding problem over a field $K$ is an epimorphism

$$
\beta: B \rightarrow \mathcal{G}(L / K)
$$

where $L / K$ is a finite Galois extension and $B$ is a finite group. If $K^{\prime}$ is a regular extension of $K$ and $L^{\prime}=L K^{\prime}$, then restriction $\rho=\operatorname{res}_{L^{\prime} / L}: \mathcal{G}\left(L^{\prime} / K^{\prime}\right) \rightarrow \mathcal{G}(L / K)$ is an isomorphism. So, $\rho^{-1} \circ \beta: B \rightarrow \mathcal{G}\left(L^{\prime} / K^{\prime}\right)$ is an embedding problem over $K^{\prime}$. A solution of $\beta$ over $K^{\prime}$ is an isomorphism $\gamma: \mathcal{G}\left(M^{\prime} / K^{\prime}\right) \rightarrow B$, where $M^{\prime}$ is 
a Galois extension of $K^{\prime}$ which contains $L^{\prime}$ such that $\beta \circ \gamma=\operatorname{res}_{M^{\prime} / L}$. We call $M^{\prime}$ a solution field of $\beta$ over $K^{\prime}$.
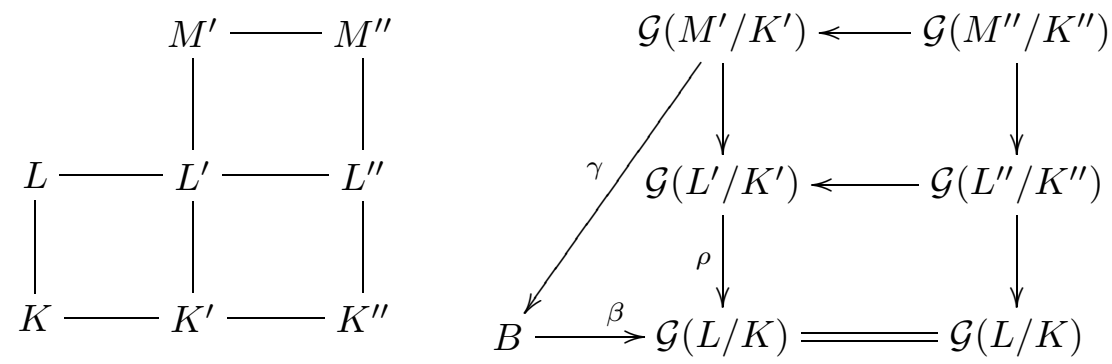

If $K^{\prime \prime}$ is a regular extension of $K^{\prime}, L^{\prime \prime}=L K^{\prime \prime}$, and $M^{\prime \prime}=M^{\prime} K^{\prime \prime}$, then $\gamma \circ \operatorname{res}_{M^{\prime \prime} / M^{\prime}}$ is a solution of $\beta$ over $K^{\prime \prime}$.

Finally, let $\beta_{i}: B_{i} \rightarrow \mathcal{G}\left(L_{i} / K\right), i=1, \ldots, n$ be finite embedding problems over $K$. Construct the compositum $L=L_{1} \cdots L_{n}$ and the fiber product

$B=\left\{\left(b_{1}, \ldots, b_{n}, \sigma\right) \in B_{1} \times \cdots \times B_{n} \times \mathcal{G}(L / K) \mid \beta_{i}\left(b_{i}\right)=\operatorname{res}_{L / L_{i}} \sigma, i=1, \ldots, n\right\}$

Let $\beta: B \rightarrow \mathcal{G}(L / K)$ be the projection on the last coordinate and for each $i$ between 1 and $n$ let $\pi_{i}: B \rightarrow B_{i}$ be the projection on the $i$ th coordinate. Observe that both $\pi_{i}$ and $\beta$ are surjective. So, $\beta$ is an embedding problem over $K$ which dominates each of the problems $\beta_{i}$. Let $\gamma: \mathcal{G}(M / K) \rightarrow B$ be a solution of $\beta$. Let $M_{i}$ be the fixed field in $M$ of $\operatorname{Ker}\left(\pi_{i} \circ \gamma\right)$ and let $\gamma_{i}: \mathcal{G}\left(M_{i} / K\right) \rightarrow B_{i}$ be the isomorphism induced by $\pi_{i} \circ \gamma$. Then $\gamma_{i}$ is a solution of $\beta_{i}, i=1, \ldots, n$.

Lemma 2.4. Let $\beta: B \rightarrow \mathcal{G}(L / K)$ be a finite embedding problem over $K$. Then $K$ has a finitely generated regular extension $K^{\prime}$ over which $\beta$ has a solution $\gamma: \mathcal{G}\left(M^{\prime} / K^{\prime}\right) \rightarrow B$ such that $M^{\prime}$ is a finitely generated purely transcendental extension of $L$.

Proof. Choose a set $\left\{x^{b} \mid b \in B\right\}$ of algebraically independent elements over $K$ labeled by the elements of $B$. Construct the purely transcendental extension $M^{\prime}=L\left(x^{b} \mid b \in B\right)$ of $L$. Let $B$ act on $M^{\prime}$ by the following rule: $a^{b^{\prime}}=a^{\beta\left(b^{\prime}\right)}$ and $\left(x^{b}\right)^{b^{\prime}}=x^{b b^{\prime}}$ for $a \in L$ and $b, b^{\prime} \in B$. Denote the fixed field of $B$ under this action by $K^{\prime}$. Let $L^{\prime}=L K^{\prime}$. By Galois theory, $M^{\prime} / K^{\prime}$ is a Galois extension with Galois group $B$. Moreover, the identification of $\mathcal{G}\left(M^{\prime} / K^{\prime}\right)$ with $B$ is a solution of $\beta$ over $K$. In particular, $L \cap K^{\prime}=K$. Since $L^{\prime} / L$ is a subextension of a purely transcendental extension, it is regular. Hence, $K^{\prime} / K$ is also a regular extension. Finally, by [Lan2, p. 64], $K^{\prime} / K$ is finitely generated.

Denote the free profinite group of rank $\aleph_{0}$ by $\hat{F}_{\omega}$. If $K$ is a countable field over which every finite embedding problem is solvable, then, by Iwasawa's criterion, $G(K) \cong \hat{F}_{\omega}$ [FrJ, Cor. 24.2]. 
Lemma 2.5. Every countable field $K_{0}$ has a regular countable extension $K$ with the following properties:

(a) $K$ is weakly PAC.

(b) $C(K)=C\left(K_{0}\right)$ for each curve $C$ over $K_{0}$ with genus $(\tilde{C}) \geq 2$.

(c) $K$ is Hilbertian.

(d) $G(K) \cong \hat{F}_{\omega}$.

Proof. By induction we construct an ascending sequence $K_{0}=F_{0} \subset F_{1} \subset F_{2} \subset$ ... of countable fields. For each $m$ we list the varieties over $F_{m}$ which are either of type 0 or of type 1 as $V_{m 1}, V_{m 2}, V_{m 3}, \ldots$ Also, we list the finite embedding problems over $F_{m}$ as $\beta_{m 1}, \beta_{m 2}, \beta_{m 3}, \ldots$. These objects should have the following properties:

(2a) $F_{m+1}$ is the function field over $F_{m}$ of a direct product of varieties which are defined over $F_{m}$ and are either of type $0^{\prime}$ or of type 1.

(2b) $V_{i j}\left(F_{m+1}\right) \neq \emptyset$ for $1 \leq i, j \leq m$.

(2c) $\beta_{i j}$ has a solution over $F_{m+1}$ for $1 \leq i, j \leq m$.

Indeed, if $F_{m}$ has already been constructed, we choose an embedding problem $\beta$ over $F_{m}$ which dominates $\beta_{i j}$ for $1 \leq i, j \leq m$. Lemma 2.4 gives a finitely generated regular extension $F_{m}^{\prime}$ of $F_{m}$ over which $\beta$ is solvable. Moreover, $F_{m}^{\prime}$ is contained in a finitely generated purely transcendental extension of $\tilde{F}_{m}$. Thus, $F_{m}^{\prime}$ is the function field of a variety $U$ of type $0^{\prime}$ over $F_{m}$.

Next let $V=\prod_{i, j=1}^{m} V_{i j} \times_{F_{i}} F_{m}$, choose a generic point $\mathbf{x}$ for $V \times_{F_{m}} F_{m}^{\prime}$ and let $F_{m+1}=F_{m}(\mathbf{x})$. Then $F_{m+1}$ is a finitely generated regular extension of $F_{m}^{\prime}$, therefore also of $F_{m}$. Moreover, $F_{m+1}$ is the function field of $U \times V$. The projection of $\mathbf{x}$ on the $i j$ th coordinate is an $F_{m+1}$-rational point of $V_{i j}$, $1 \leq i, j \leq m$. Finally, $\beta_{i j}$ has a solution over $F_{m}$ for $1 \leq i, j \leq m$.

The union $K$ of all $F_{i}$ is a countable regular extension of $K_{0}$. If $W$ is a variety of type 0 or of type 1 over $K$, then there exist $i$ and $j$ such that $W \cong V_{i j} \times_{F_{i}} K$. Let $m=\max (i, j)$. By $(2 \mathrm{~b}), V_{i j}\left(F_{m+1}\right) \neq \emptyset$. Hence, $W(K) \neq \emptyset$. Conclude that $K$ is weakly PAC.

If $\beta: B \rightarrow \mathcal{G}(L / K)$ is a finite embedding problem, then it is induced by $\beta_{i j}$ for some $i$ and $j$. Again, let $m=\max (i, j)$. By (2c), $\beta_{i j}$ is solvable over $F_{m+1}$. Hence, $\beta_{i j}$ and therefore $\beta$ is solvable over $K$. Conclude from Iwasawa's criterion that $G(K) \cong \hat{F}_{\omega}$.

By (2a) and by [FrJ, Thm. 12.10] each $F_{m}$ is Hilbertian. Conclude from Lemma 2.3 that $K$ is Hilbertian.

Finally, let $C$ be a curve over $K_{0}$ with genus $(\tilde{C}) \geq 2$. By (2a) and by Corollary 2.2, $C\left(F_{m+1}\right)=C\left(F_{m}\right)$ for $m=1,2,3 \ldots$. Conclude that $C(K)=C\left(K_{0}\right)$.

Recall that a field $K$ is ample if every curve $C$ over $K$ with a simple $K$ rational point has infinitely many $K$-rational points. In particular every PAC field is ample. (For more about ample fields see [HaJ, §6].)

Theorem 2.6. Let $K_{0}$ be a finite field or a finitely generated extension of $\mathbb{Q}$. 
Then $K_{0}$ has a countable regular extension $K$ with the following properties:

(a) $K$ is weakly-PAC.

(b) $C(K)$ is finite for each curve $C$ over $K_{0}$ of genus at least 2 .

(c) $K$ is not PAC and even not ample; $K_{\text {ins }}$ is not ample.

(d) Each extension of $K_{\mathrm{ins}}$ of transcendence degree 1 satisfies the Hasse principle for Brauer groups.

(e) All Henselian closures of $K$ are separably closed.

(f) $K$ is not formally real.

(g) $K$ is Hilbertian.

(h) $G(K) \cong \hat{F}_{\omega}$.

Proof. Let $K$ be the extension of $K_{0}$ which Lemma 2.5 provides. In particular, $K$ satisfies (g) and (h). Consider a curve $C$ over $K_{0}$ of genus at least 2 . Since $K_{0}$ is perfect, genus $(\tilde{C})=\operatorname{genus}(C) \geq 2$. If $K_{0}$ is finite, then $C\left(K_{0}\right)$ is also finite. If $K_{0}$ is a finitely generated extension of $\mathbb{Q}$, then by Faltings [FaW, p. 205, Thm. 3], $C\left(K_{0}\right)$ is finite. As $C(K)=C\left(K_{0}\right)$ (by (b) of Lemma 2.5), $C(K)$ is finite. This proves (b). In particular, $K$ is not PAC [FrJ, Prop. 10.1].

Moreover, if char $\left(K_{0}\right) \neq 2,5$, the hyperelliptic curve $H$ defined over $K_{0}$ by the equation $Y^{2}=X^{5}-1$ has genus 2 [Art, p. 317]. For $\operatorname{char}\left(K_{0}\right)=5$ take $Y^{2}=X^{5}-X$, for $\operatorname{char}\left(K_{0}\right)=2$ take $Y^{2}+Y=X^{5}-1$. By (b), $H(K)$ is finite although it has a simple $K$-rational point, namely $(1,0)$. Conclude that $K$ is not ample.

If $K_{0}$ is a finite field and $(a, b) \in H\left(K_{\mathrm{ins}}\right)$, then there exists a power $q$ of $\operatorname{char}(K)$ such that $a^{q}, b^{q} \in K$. Hence $\left(a^{q}, b^{q}\right) \in H(K)=H\left(K_{0}\right)$. As $K_{0}$ is perfect, $a, b \in K_{0}$. It follows that $H\left(K_{\text {ins }}\right)$ is finite. Conclude that $K_{\text {ins }}$ is not ample.

Finally statements (d), (e), and (f) are consequences of (a), by Proposition 1.3 .

Remark 2.7. (a) The proof of Proposition 1.3(b) shows that if $K$ is a field and all Henselizations of $K_{\text {ins }}$ are separably closed, then so are all Henselizations of $K$. In particular, this is the case when $K_{\text {ins }}$ is PAC. This situation may indeed occur without that $K$ is PAC as Hrushovsky proved in [Hru]. We have therefore been careful in Theorem 2.6 to construct $K$ such that $K_{\text {ins }}$ is not PAC.

(b) An arbitrary countable field $K_{0}$ has a countable extension $K$ which satisfies (a) and (c)-(h) of Theorem 2.6. Indeed, choose a transcendental element $t$ over $K_{0}$. Then use Lemma 2.5 to find a countably generated extension $K$ of $K_{0}(t)$ such that $K$ is weakly PAC, Hilbertian, and $C(K)=C\left(K_{0}(t)\right)$ for each curve $C$ over $K_{0}(t)$ with genus $(\tilde{C}) \geq 2$. Next choose a curve $C$ over $K_{0}(t)$ with genus $(\tilde{C}) \geq 2$ such that $C\left(K_{0}(t)\right)$ is finite and has a simple point. Then (c) of Theorem 2.6 holds. Statements (d) $-(\mathrm{h})$ of Theorem 2.6 hold as in the proof of that theorem.

(c) If in the proof of Lemma 2.5 we construct the field $K$ out of $K_{0}$ by adjoining only generic points of varieties of type 0 and omit the construction 
of $F_{m}^{\prime}$, then $G(K)$ will be projective, $C(K)=C\left(K_{0}\right)$ for each curve $C$ over $K_{0}$ with genus $(\tilde{C}) \geq 1$, and $K$ will be Hilbertian and not formally real.

In view of Theorem 2.6 we may reformulate Problem 10.16(b) of [FrJ] in the following way:

Problem 2.8. Find an infinite field $K$ of a finite transcendence degree over its prime field such that $K$ is not PAC but each Henselian closure of $K$ is separably closed and $K$ is not formally real.

\section{References}

[Art] E. Artin, Algebraic Numbers and Algebraic Functions, Gordon and Breach, New York, 1967.

[Ax] J. Ax, The elementary theory of finite fields, Ann. of Math. 88 (1968), 239-271.

[Efr] I. Efrat, A Hasse principle for function fields over PAC fields, Israel J. Math. 122 (2001), 43-60.

[FaW] G. Faltings and G. Wüstholz et al., Rational Points, Seminar Bonn/Wuppertal 1983/84, Vieweg, Braunschweig 1984.

[Fre] G. Frey, Pseudo algebraically closed fields with non-archimedian real valuations, J. Algebra 26 (1973), 202-207.

[FrJ] M. D. Fried and M. Jarden, Field Arithmetic, Ergebnisse der Mathematik (3) 11 (1986), Springer-Verlag, Heidelberg.

[HaJ] D. Haran and M. Jarden, Regular split embedding problems over complete valued fields, Forum Mathematicum 10 (1998), 329-351.

[Jac] N. Jacobson, Finite Dimensional Division Algebras over Fields (1996), Springer-Verlag, Berlin.

[Hru] E. Hrushovski, A non-PAC field whose maximal purely inseparable extension is PAC, Israel J. Math 85 (1994), 199-202.

[Lan1] S. Lang, Abelian Varieties, Interscience Tracts in Pure and Applied Mathematics 7 (1959), Interscience Publishers, New York.

[Lan2] S. Lang, Introduction to algebraic geometry, Interscience Publishers, New York, 1958.

[LaT] S. Lang and J. Tate, Principal Homogeneous spaces over abelian varieties, Amer. J. Math. 80, 659-684.

[Rib] P. Ribenboim, Théorie des valuations (1964.), Les Presses de l'Université de Montréal, Montréal.

[Sch] A. Schinzel, Selected Topics on Polynomials, The University of Michigan Press, 1982.

[Ser] J.-P. Serre, Corps locaux, Actualités scientifiques et industrielles 1296 (1968), Hermann, Paris.

Mathematisches Institut, Universität Erlangen-Nürnberg, Bismrackstrasse $1 \frac{1}{2}$, Erlangen 91054, Germany.

E-mail address: geyer@mi.uni-erlangen.de

School of Mathematics, Tel Aviv University, Ramat Aviv, Tel Aviv 69978, Israel.

E-mail address: jarden@post.tau.ac.il 